\title{
Percepción de profesionales de la inclusión socioeducativa sobre programas destinados a jóvenes vulnerables
}

Enviado: 22 de septiembre de 2021 / Aceptado: 23 de noviembre de 2021 / Publicado: 31 de diciembre de 2021

FRANCISCO JOSÉ GARCÍA AGUILERA Departamento Didáctica General y Organización Escolar, Universidad Internacional de La Rioja, España francisco.garcia.a@unir.ne (D) $0000-0002-5764-7409$

JUAN JOSÉ LEIVA OLIVENCIA Departamento Didáctica y Organización Escolar, Universidad de Málaga, España juanleiva@uma.es

DIEGO AGUILAR CUENCA

Instituto Municipal de Formación y Empleo del Ayuntamiento de Málaga, España daguilar@malaga.eu (iD) $0000-0001-5336-2687$

\section{RESUMEN}

El objetivo de este estudio ha sido conocer las percepciones de profesionales españoles de la inclusión socioeducativa acerca de programas formativos dirigidos a jóvenes de entre 16 y 29 años con el propósito de evidenciar la realidad de los mismos, las competencias técnicas más trabajadas con los usuarios y las áreas de mejora de dichos programas. Se ha llevado a cabo un diseño de encuesta, en el que participaron 120 profesionales. Se ha procedido a realizar un análisis descriptivo, en el marco de un estudio no experimental. Los resultados muestran que programas financiados con fondos públicos son valorados con menor puntuación por su baja efectividad en materia

\section{ABSTRACT}

Perception of professionals of socio-educational inclusion on programs aimed at vulnerable youth

The objective of this study has been to know the perceptions of spanish professionals of socio-educational inclusion about training programs aimed at young people between 16 and 29 years old to demonstrate their reality, the technical skills most worked with users and areas for improvement of these programs. A survey design has been carried out in which 120 professionals participated. A descriptive analysis has been carried out, within the framework of a nonexperimental study. The results show that programs 
de inclusión socioeducativa, mientras que iniciativas de inserción sociolaboral relacionadas con el fomento de competencias técnicas o los programas de colaboración público-privada obtienen una valoración más positiva al contemplar un fortalecimiento de las competencias relacionales y las TIC.

Palabras Clave: Inclusión, jóvenes, contextos socioeconómicos desafiantes, formación, vulnerabilidad. financed with public funds are valued with a lower score due to their low effectiveness in terms of socio-educational inclusion, while socio-labour insertion initiatives related to the promotion of technical skills or public-private collaboration programs obtain an assessment more positive when contemplating a strengthening of relational competences and ICT.

Keywords: Inclusion, young people, challenging socio-economic contexts, training, vulnerability.

\section{INTRODUCCIÓN}

Los programas de inclusión socioeducativa suponen una vía alternativa a los sistemas reglados de educación, puesto que, a través de procesos de educación no formal, los jóvenes que se benefician de dichas iniciativas logran obtener una segunda oportunidad educativa y conseguir una inclusión a nivel socioeducativo, así como una incorporación guiada al mercado laboral (Salva, Nadal \& Agnés, 2017; Olmos \& Más, 2018; García, 2020).

La Agenda 2030 (2015) ha sido decisiva para formular el Objetivo de Desarrollo Sostenible 4 (en adelante ODS 4), hace referencia a la educación y trata de ofrecer garantías para una inclusión educativa y de calidad y ofrecer oportunidades de aprendizaje permanente para todas las personas. La Agenda se inspira en un planteamiento de la educación y del desarrollo basado en la justicia social, la dignidad, y la protección integral de los derechos humano.

El Informe de Seguimiento de la Educación en el Mundo 2020 "Inclusión y Educación", ofrece a los gobernantes encargados de formular políticas una visión exhaustiva para realizar un seguimiento hacia conseguir que se cumpla dicho ODS 4, basándose en sus elementos y metas claves y evaluando el análisis de indicadores y resultados que se van obteniendo en materia de educación e inclusión. Dicho Informe, plantea como uno de sus mensajes clave "abordar la inclusión en la educación, haciendo una llamada de atención sobre todas las personas que son excluidas de la educación, debido a sus antecedentes o habilidades” (p.1).

Los sistemas educativos se organizan de forma lineal, en ellos, el alumnado tiene oportunidades limitadas para concluir la educación básica obligatoria, pasando de un nivel educativo 
a otro. Esta organización debe replantearse, sobre todo a raíz del concepto de aprendizaje a lo largo de la vida, que supone uno de los principales baluartes del desarrollo de las personas:

[...] En relación con la organización de las enseñanzas y el aprendizaje a lo largo de la vida, se añaden unas precisiones sobre la educación básica, con el fin de garantizar la continuidad, coordinación y cohesión entre las dos etapas que la componen y de resaltar que su finalidad consiste en universalizar los conocimientos y las competencias que permitan aprender a todos los alumnos y alumnas a aprender a lo largo de la vida y faciliten su plena participación social y laboral [...] (Ley Orgánica 3/2020, de 29 de diciembre, por la que se modifica la Ley Orgánica 2/2006, de 3 de mayo, de Educación: 122873).

Es en el año 1995 cuando la Comisión Europea utiliza el término de "Escuela de Segunda Oportunidad" como programas alternativos con un fin inclusivo y socioeducativo dirigido específicamente a la población joven de entre 16 y 29 años de edad. Se pone entonces de manifiesto que en los países miembros y en las distintas ciudades europeas, hay un creciente número de jóvenes que no poseen los niveles mínimos de cualificación aportados por la enseñanza básica, debido a su abandono escolar temprano o a las elevadas cifras de fracaso escolar; acompañado todo ello por unas condiciones socioeconómicas precarias debido a su procedencia de contextos en situación de vulnerabilidad. En esa misma fecha, la Comisión Europea publica el Libro Blanco sobre la Educación y la Formación: Enseñar y Aprender. Hacia una Sociedad cognitiva (Mills \& Mc Gregor, 2010; Escarbajal, Izquierdo \& López, 2013; Corchuelo, Aránzazu, González \& Morón, 2016; Eurofound and the International Labour Office, 2017).

Como parte fundamental de estos programas, cobran una especial relevancia las personas que trabajan en ellos: personas voluntarias, personal técnico, gestores y directivos. Dichos profesionales conocen de primera mano la implementación de las políticas, planes y programas que se llevan a cabo en materia de apoyo a la inclusión socioeducativa de personas que se encuentran en situación vulnerable. Iniciativas como los Talleres de Empleo, el Programa Experiencias Profesionales para el Empleo, el Programa Operativo de Empleo, Formación y Educación o las Escuelas de Segunda Oportunidad Educativa son un ejemplo de iniciativas que se están desarrollando en nuestro país y que los técnicos que las llevan a cabo conocen de primera mano en cuanto a su alcance, implementación y evaluación de los resultados que se obtienen a nivel de inclusión socioeducativa de las personas beneficiarias que participan en dichas iniciativas (Herrera, 2014; Olmos, 2014; Prieto, 2015; Salva, Nadal \& Agnés, 2017; Olmos \& Más, 2018; García 2019; Martíns, Carneiro, Campos, Mota, Negrão, Baptista \& Matos, 2020). 
Este trabajo, ofrece la visión integral desde la perspectiva de los profesionales aportando una contribución de gran valor en lo relativo a la situación actual de los programas de inclusión socioeducativa junto a su percepción crítica en cuanto a las mejoras necesarias a implementar para ofrecer una respuesta más adaptada a las necesidades socioeducativas de los jóvenes más vulnerables.

Diferentes investigaciones y estudios han establecido el marco donde se inscriben las experiencias educativas de segunda oportunidad, a través de diferentes dispositivos que diseñan acciones más flexibles y diversificadas ofreciendo opciones y oportunidades adaptadas para cubrir las competencias clave, basadas en los elementos aparecidos en la Recomendación del Parlamento Europeo y del Consejo, de 18 de diciembre de 2006 relativa a las competencias clave para el aprendizaje permanente (2006/962/CE), y que se completan con formación práctica en centros de trabajo, ofreciendo de este modo una segunda oportunidad educativa (Olmos, 2014; Prieto, 2015; Salva, Nadal \& Agnés, 2017; Vázquez \& Barrera, 2017; Olmos \& Más, 2018; Martíns, Carneiro, Campos, Mota, Negrão, Baptista \& Matos, 2020; García 2020; García, Medina, Amashta \& Higueras, 2021).

El Programa Operativo de Empleo, Formación y Educación del Fondo Social Europeo (2013: 9-10) para el período 2014-2020 diseña un plan de acciones formativas centradas en itinerarios de inserción sociolaboral y certificados de profesionalidad que permitan un acceso cualificado a dicha población juvenil. Dichas actuaciones, parten de una situación compleja a nivel de la población general y aún más delicada para los jóvenes:

\footnotetext{
"Si bien la tasa de abandono educativo temprano en España ha disminuido de forma continuada desde 2008 y se ha situado por debajo del $25 \%$ a partir del 2012, se deben atajar las causas subyacentes y sentar las bases para la mejora del nivel educativo de la población mayor de 25 años [...]. Existen dos importantes factores de abandono: el rendimiento escolar (a menor rendimiento, mayor probabilidad de abandono) y la tasa de paro juvenil (el incremento de la misma disuade al abandono prematuro). No terminar con éxito la enseñanza obligatoria es el principal factor de abandono".
}

Para el período 2021-2027 y partiendo del documento de la nueva estrategia FSE+ se conforma por tres recursos del período 2014-2020: el Fondo Social Europeo (FSE), la Iniciativa de Empleo Juvenil (IEJ) y el Fondo de Ayuda Europea para las Personas Más Desfavorecidas (FEAD), todo ello orientado a programas específicos que aludan a actuaciones que apoyan la inclusión socioeducativa de los jóvenes. Para lograr estos objetivos se busca reducir la repe- 
tición de curso académico, el fracaso escolar y el abandono temprano, mejorar el sistema de Formación Profesional del Sistema Educativo respondiendo a las profesiones y sectores más demandados y facilitando su acceso, optimizar las relaciones entre educación y empleo, así como favorecer la transición entre los diferentes niveles educativos "especialmente en atención a las personas jóvenes que ni estudien, ni trabajen ni reciben formación" (Nueva Estrategia FSE+, 2020, Ministerio de Trabajo y Economía Social, p. 8).

Iniciativas de colaboración público-privada como las Lanzaderas de Empleo promovidas por Fundación Santa María La Real, Vives Emplea, programa llevado a cabo por Acción contra el Hambre con la colaboración del Fondo Social Europeo o el Programa Incorpora de la Fundación de la Obra Social "La Caixa", todas ellas iniciativas centradas en la inclusión socioeducativa y laboral de personas jóvenes en situación vulnerable (Longás, De Querol, Ciraso, Rierad \& Úcar, 2018; Gómez, 2019).

Estos programas están conformados por jóvenes socialmente marginados que abandonaron el sistema educativo y que ya no están participando de la enseñanza obligatoria. Además, no disponen de la cualificación, ni de las habilidades necesarias para hallar un empleo, o bien para continuar con algún programa de formación específica para acceder al mercado laboral. Queda más que demostrado que se tiene mayor dificultad de encontrar un empleo cuando no se dispone de una titulación oficial, como consecuencia de un abandono prematuro o ante un fracaso escolar. Generalmente, tras ese abandono, este colectivo se ve abocado a trabajos de corta duración y poca calidad, situaciones de dificultad, posible delincuencia y diferentes situaciones de adicción en el peor de los casos (Vázquez, 2015; Prieto, 2015; Salva, Nadal, Meliá, 2016; Olmos \& Más, 2018, García, 2020; Leiva et al. 2020).

Por tanto, existe una necesidad inminente de ofrecer oportunidades a estas personas para la vuelta al proceso de educación a lo largo de la vida trabajando competencias sociolaborales, que la Comisión Europea (2018) identifica entre sus competencias clave para este fin y que suponen uno de los principales propósitos de estos programas de inclusión socioeducativa, lo que permitirá a estos jóvenes garantizar su inclusión a corto-medio plazo (Domínguez, 2019).

Después de los años de crisis económica global desde 2007 y tras la pandemia mundial ocasionada por la COVID-19, nos situamos en un panorama complicado que nos ha afectado de forma directa en todas las dimensiones personales y profesionales, impactando de manera más directa a personas en situación vulnerable entre ellas los jóvenes, alcanzando a colectivos 
anteriormente ya marginados y ampliando sus efectos a los ámbitos sanitario, socioeconómico, cultural y por supuesto educativo, alcanzando a miles y miles de personas por todo el mundo (Ponce de León, 2020).

En este contexto precario, y con muchas dificultades de acceso a la formación y al empleo con niveles de desempleo elevados, problemas de acceso a la vivienda y recursos limitados en los servicios públicos, etc., aumentan los casos de exclusión social, laboral y educativa, ante los que las instituciones comunitarias, nacionales y regionales, no deben quedarse ajenos. Esta situación ha de ser atendida desde diversas dimensiones y por los diferentes agentes sociocomunitarios (Ainscow, Booth \& Dyson, 2006, Corchuelo, Aránzazu, González \& Morón, 2016, Mcmillan, Rodrik, \& Sepúlveda, 2017, Aguilera \& Caballero, 2019; García, 2019; European Comission, 2020).

En la actualidad, y teniendo en cuenta la situación provocada por la COVID 19 y en mayor medida en las personas jóvenes, se trabaja a partir de un documento denominado Sobre un marco para la cooperación europea en el ámbito de la juventud: la Estrategia de la Unión Europea para la Juventud 2019-2027, con una serie de objetivos prioritarios y que han sido matizados tras el impacto de la pandemia contemplando los siguientes aspectos: llevar a cabo la inclusión de jóvenes en situación vulnerable, ofrecer garantías para el acceso de los jóvenes a la formación y al mundo laboral así como promocionar y reconocer también las vías de educación no formal y su aprendizaje. Así mismo, este documento respalda la transición de estos jóvenes del ámbito escolar al mundo laboral (Arredondo \& Vizcaíno, 2020).

El objetivo de este estudio ha sido conocer las percepciones de profesionales de la inclusión socioeducativa acerca de programas formativos y de fomento de la empleabilidad dirigidos a jóvenes de entre 16 y 29 años con el propósito de evidenciar la realidad de los mismos y sus áreas de mejora. De forma más concreta, planteamos los siguientes objetivos específicos:

- Conocer los diferentes programas e iniciativas existentes que promueven la inclusión socioeducativa de jóvenes en situación vulnerable.

- Identificar las competencias técnicas más trabajadas con los usuarios de estos programas para favorecer su inclusión socioeducativa.

- Identificar la efectividad de los programas en materia de inclusión socioeducativa y sus áreas de mejora. 


\section{METODOLOGÍA}

\subsection{Diseño}

En nuestro estudio, se utilizó una metodología esencialmente cuantitativa, no experimental, con el propósito de representar la realidad existente en España en relación con los programas de inclusión socioeducativa de jóvenes en situación vulnerable. Específicamente es un estudio de encuesta. Para la recogida de información, se diseñó un cuestionario ad hoc con cuestiones cerradas, redactadas en forma de escala de calificación y tipo Likert. También se incluyeron preguntas cortas que ayudaron a los encuestados a ajustar algunas de las respuestas realizadas. A partir de los resultados obtenidos del cuestionario se ha realizado un análisis descriptivo de las variables objeto de estudio.

\subsection{Participantes}

En este estudio participaron un total de 120 profesionales de la inclusión socioeducativa (91 mujeres y 29 hombres). El contexto en el que se implementó la encuesta fue en el de los programas de inclusión socioeducativa dirigidos principalmente a jóvenes en situación vulnerable desarrollados a nivel nacional. Los profesionales encuestados pertenecían al sector público (28,3\%), empresas privadas $(4,2 \%)$ y al tercer sector social -fundaciones, asociaciones y ONG- $(67,5 \%)$.

\subsection{Instrumento de recogida de datos}

Como herramienta para la recogida de datos, se ha confeccionado un cuestionario ad hoc para este estudio partiendo de una revisión bibliográfica exhaustiva sobre el tema (Vázquez, 2015; Prieto, 2015; Salva, Nadal \& Meliá, 2016; Olmos \& Más, 2018, García, 2020).

El cuestionario denominado "Percepción de los profesionales de la inclusión socioeducativa sobre programas destinados a jóvenes en riesgo de vulnerabilidad" tiene el objetivo de indagar acerca de las necesidades de formación de los profesionales que forman parte de organismos y entidades sociales. De este modo, la formulación de cada ítem cumple los objetivos que persigue, señalando el investigador mediante esta definición de objetivos, la información que necesita. De este modo, y siguiendo a Ruiz (2002), los ítems planteados han sido elaborados de forma sencilla, siendo las preguntas formuladas coherentes con el tema de investigación. También se ha tenido en cuenta no emplear ítems negativos y términos confusos, tal y como se muestra en la tabla 1. 
Tabla 1. Muestra de pr egunt as extraídas del Cuestionario "P ercepción de los pr ofesionales de la inclusión socioeducativ a sobre programas destinados a jó venes en riesgo de vulner abilidad". Fuente. Elaboración propia

\begin{tabular}{l} 
Datos sociodemogr áficos: Géne o | Edad | Niv el formativo | Titulación | Puest o de trabajo actual \\
Valora el grado de efectividad de los siguientes programas para la inser ción de personas desempleadas. (1: menor puntuación \\
y 10: mayor puntuación) \\
\hline POEFE (Programa Oper ativo de Emple o, Formación y Educación) \\
EDUSI (Estrategia de Desarr ollo Urb ano Sostenible e Int egrado) \\
¿Qué tipo de compet encias sociolabor ales trabajas más con tus usuarios/as? (1: menor puntuación y 10: may or puntuación) \\
Planificación del tiemp \\
Búsqueda de emple o (orient ación y diseño del currículum) \\
¿Qué recursos públicos deberían reforzarse para mejorar la empleabilidad de personas desempleadas de larga duración? (1: \\
menor puntuación y 10: may or puntuación) \\
Agencias de colocación \\
Ayudas al empr endimient o \\
¿En qué grado te parecen una innovación en el ámbito de la inclusión sociolabor al los siguientes recursos para el empleo? (1: \\
menor puntuación y 10: may or puntuación) \\
Lanzader as de emple o \\
Vives Emplea
\end{tabular}

¿Qué nivel de efectividad tienen los siguientes recursos relacionados con las Tecnologías de la Información y la Comunicación en la mejor a de la emple abilidad?

Aula Ment or

Centro de ac ceso público a int ernet

Según tu experiencia ¿Qué recursos de inclusión social podrían ser más efectivos frente a la situación actual ocasionada por la COVID19?

\subsection{Procedimiento}

La muestra fue de tipo no probabilístico incidental. Las encuestas fueron enviadas a través de un mensaje de participación a alrededor de 150 profesionales a nivel nacional que al menos contaran con 3 años de experiencia como técnicos en el tipo de programas socioeducativos objeto del presente estudio, facilitándoles un enlace a formularios de Google, tras haberles proporcionado información sobre el objetivo de la investigación. Finalmente cumplimentaron el cuestionario 
120 profesionales del ámbito de los programas de inclusión socioeducativa procedentes del sector público y privado a nivel nacional (administración pública, fundaciones, asociaciones y ONG).

Para evaluar la fiabilidad se empleó el método de consistencia interna coeficiente Alfa de Cronbach, obteniendo un valor de.883. Para comprobar la validez de contenido del instrumento se llevó a cabo una valoración por jueces a través de 8 expertos en investigación educativa que hubieran participado en proyectos de investigación relacionados con programas de inclusión socioeducativa con personas jóvenes vulnerables, con el objetivo de identificar posibles errores o sesgos en la redacción de las preguntas o dificultades de comprensión e interpretación, efectuando posteriormente las oportunas rectificaciones y mejoras de algunos de estos ítems.

Previamente a la realización del análisis factorial de componentes principales obtuvimos información sobre las medidas referidas al cumplimiento de los criterios de aplicación de dicho análisis:

1. Kaiser-Meyer-Olkin (KMO) que aporto datos sobre la adecuación del muestreo. El valor obtenido en $\mathrm{KMO}$, fue .778. Lo que indica que el análisis factorial es práctico y útil para este estudio.

2. A través de la Prueba de esfericidad de Barlett podemos concluir que la significación es adecuada, ya que se obtiene un valor inferior a 0.00005 , por lo que se puede rechazar la hipótesis nula, considerando el ajuste de las variables mediante el análisis factorial idóneo.

Tabla 2. Indicador es KMO y prueb a de Bartlett. Fuente. Elabor ación pr opia

\begin{tabular}{lll} 
Medida de adecuación muestr al de Kaiser-Mey er-Olkin (KMO) & .778 \\
\hline Chi-cuadr ado apr oximado & 1737.431 \\
\cline { 2 - 3 } Prueba de esf ericidad de Bartlett & Grados de libert ad & 378 \\
\cline { 2 - 3 } & Significación (menor que & .000
\end{tabular}

Una vez realizado el análisis de componentes principales, se obtuvieron 3 factores. Dichos componentes (autovalores $\leq 1$ ) explican el $70.925 \%$ de la varianza total. 
Tabla 3. Análisis de component es princip ales. Fuente. Elabor ación pr opia

\begin{tabular}{|cccc}
\hline \multirow{2}{*}{ Componente } & \multicolumn{3}{c}{ Sumas de rotación de cargas al cuadrado } \\
\cline { 2 - 4 } & Total & \% de varianza & \% acumulado \\
\hline $\mathbf{1}$ & 4.043 & 14.440 & 14.440 \\
\hline $\mathbf{2}$ & 3.164 & 11.301 & 25.741 \\
\hline $\mathbf{3}$ & 2.407 & 8.598 & 34.338 \\
\hline $\mathbf{4}$ & 2.396 & 8.558 & 42.896 \\
\hline $\mathbf{5}$ & 2.289 & 8.174 & 51.070 \\
\hline $\mathbf{6}$ & 2.260 & 8.071 & 59.141 \\
\hline $\mathbf{7}$ & 1.896 & 6.772 & 65.912 \\
\hline $\mathbf{8}$ & 1.403 & 5.012 & 70.925 \\
\hline
\end{tabular}

Además, se llevó a cabo un Análisis de Componentes Principales, a través de una rotación de normalización Varimax con Kaiser, que convergió en 8 iteraciones. Para ello se ha utilizado la aplicación SPSS v.25. La reagrupación de factores quedó como muestra la tabla 4.

Tabla 4. Resultados del análisis de component es princip ales. Reagrupación de f actores. Fuente. Elabor ación propia

Factor 1. Programas de inclusión socioeducativa financiados con fondos públicos (cofinanciación europea)

\begin{tabular}{lc} 
Ítem & Carga factorial \\
\hline Experiencias Pr ofesionales p ara el Emple o (EPES) - Prácticas pr ofesionales no labor ales & .611 \\
\hline Orient ación labor al & .590 \\
\hline Escuelas de Segunda Oportunidad Educativ a & .587 \\
\hline $\begin{array}{l}\text { Estrategia de Desarrollo Urbano Sostenible e Integrado (EDUSI) - Acciones para prevenir la pobreza y y } \\
\text { favorecer la inclusión social }\end{array}$ & .577 \\
\hline Formación Pr ofesional para el Empleo - Certificados de p ofesionalidad & .526
\end{tabular}

Programa Oper ativo de Emple o, Formación y Educación (POEFE) - Pr ogramas formativos 


\begin{tabular}{|c|c|}
\hline Centros de conexión pública a Int ernet & .428 \\
\hline Talleres de Emple o & .388 \\
\hline \multicolumn{2}{|l|}{ Factor 2. Programas de mejora de competencias sociolaborales para la empleabilidad } \\
\hline Habilidades r elacionales (comunicación y tr abajo en equipo) & .625 \\
\hline Búsqueda de emple o (orient ación y diseño del currículum) & .486 \\
\hline Mejora de las compet encias TIC & .466 \\
\hline Autoconocimient o y motiv ación personal & .428 \\
\hline Planificación del tiemp & .332 \\
\hline \multicolumn{2}{|l|}{ Factor 3. Programas socioeducativos con financiación público-privada } \\
\hline $\begin{array}{l}\text { Lanzader as de empleo - Búsqueda de trabajo en equipo de forma personalizada (Fundación Santa María } \\
\text { La Real) }\end{array}$ & .574 \\
\hline Vives Emplea (Acción contr a el Hambr e) & .518 \\
\hline Itiner arios de Inser ción Sociolabor al & .506 \\
\hline Agencias de colocación & .498 \\
\hline Programa Incorpor a - Itiner arios personalizados de inser ción (Obr a Social "La Caixa" & .486 \\
\hline Ayudas al empr endimient o & .452 \\
\hline Aula Ment or & .327 \\
\hline
\end{tabular}

El factor 1 es el que más ítems incluye. Se ha denominado "Programas de inclusión socioeducativa financiados con fondos públicos (cofinanciación europea)”. En dicho factor se incluyen aquellos programas de inclusión socioeducativa financiados con fondos públicos y que están directamente relacionados con la formación, el empleo y las prácticas profesionales no laborales en empresas. En el factor 2 "Programas de mejora de competencias sociolaborales para la empleabilidad" se observa la importancia crucial que los encuestados confieren a las habilidades relacionales como la comunicación y el trabajo en equipo, así como la mejora de estrategias de búsqueda de empleo, entre otras. Por último, los "Programas socioeducativos 
con financiación público-privada" se ubican en el factor 3, con una carga factorial muy significativa, y en los que destacan aquellos programas más novedosos y que se llevan a cabo a través de la iniciativa de cofinanciación público-privada.

\section{RESULTADOS}

\subsection{Datos sociodemográficos}

En nuestro trabajo, se ha podido demostrar que las personas que trabajan en el tercer sector configuran un perfil de empleo muy cualificado, siendo el $75.8 \%$ mujeres y el $24.2 \%$ hombres, con la particularidad que en su mayoría son personas mayores de 44 años (48.3\%). Con respecto a la formación académica de las personas encuestadas, en su mayoría han realizado estudios superiores universitarios $(90.6 \%)$ en titulaciones relacionadas con el ámbito de las Ciencias Sociales y Jurídicas (Pedagogía, Educación Social, Psicología, Trabajo Social, Graduado/a en Maestro/a en sus diferentes especialidades) desarrollando su actividad profesional en su mayoría en Fundaciones (49.2 \%), Entidades públicas (Ayuntamientos o Diputaciones Provinciales) (28.3\%), ONG (13.3\%) y Asociaciones (5 \%).

En lo que respecta a las áreas de actividad, los profesionales encuestados trabajan en los siguientes puestos de trabajo: el $38.3 \%$ se trata de personal técnico del área de empleo, el $26.7 \%$ trabaja en el área de orientación laboral, el $14.2 \%$ son personal de apoyo a programas, un $10.8 \%$ lo hace en el ámbito de la formación y el 10 \% restante es personal técnico de intermediación laboral.

Por último, y como datos referidos al perfil sociodemográficos del colectivo de profesionales encuestados, el $65 \%$ posee más de 7 años de experiencia en el ámbito de la inclusión socioeducativa, el $15.8 \%$, son aquellos que cuentan con menos de 3 años de experiencia en el sector, seguidos de aquellos profesionales que poseen entre 3 y 5 años de experiencia (11.7\%), a continuación, tendríamos el segmento de experiencia entre 5 y 7 años en el que se ubican el $7.5 \%$ de los encuestados.

\subsection{Resultados de los ítems de la encuesta}

Teniendo en cuenta el conjunto de ítems que se valoran en el factor 1 referidos a los programas educativos financiados con fondos públicos, los resultados obtenidos señalan como aquellos más efectivos para la inclusión socioeducativa de jóvenes vulnerables son la Formación Profesional para el Empleo a través de los certificados de profesionalidad subvencionados por 
el Fondo Social Europeo con una puntuación de 8.12, le siguen en cuanto a grado de efectividad, los Talleres de Empleo valorados con un 7.93, tras ellos el Programa EPES (Experiencias Profesionales para el Empleo) de prácticas profesionales no laborales en empresas valorado con un 7.52, seguido de los Centros de conexión pública a Internet con una puntuación de 7.45. Las denominadas como Escuelas de Segunda Oportunidad Educativa obtienen una puntuación de 7.42, muy por delante de los programas formativos del POEFE (Programa Operativo de Empleo, Formación y Educación) valorados por su efectividad en la inclusión socioeducativa con un 5.93, situando como la iniciativa más deficitario en cuanto a su efectividad en la inclusión de personas jóvenes vulnerables, el programa EDUSI (Estrategias de Desarrollo Urbano Sostenible) siendo consideradas con una muy baja puntuación (5.74) sus acciones para prevenir la pobreza y promover la inclusión social. Queda de manifiesto, la necesidad de mejorar algunos programas socioeducativos sostenidos con fondos públicos, cofinanciados con fondos europeos, y que poseen una trayectoria destacada, pero que, a juicio de los profesionales técnicos, son valorados con menor puntuación por su baja efectividad en materia de inclusión socioeducativa. En esta situación se encuentran el POEFE (Programa Operativo de Empleo, Formación y Educación) y el programa EDUSI (Estrategias de Desarrollo Urbano Sostenible) situando este último como el programa más deficitario en cuanto a su efectividad en la inclusión socioeducativa de personas jóvenes vulnerables.

El factor 2, alude a las competencias técnicas más trabajadas con los usuarios de estos programas para favorecer su inclusión socioeducativa. Los resultados fueron los siguientes partiendo desde la puntuación más alta: para las competencias "Búsqueda de empleo (orientación y diseño del currículum)" y "Autoconocimiento y motivación personal” se obtuvo una puntuación de 8.66. Para las competencias "Habilidades relacionales (comunicación y trabajo en equipo)" se otorga una valoración de 8.15 puntos, en cuanto a la "Mejora de las competencias TIC" se obtiene un promedio de 7.92 y la competencia menos trabajada en estos programas para la inclusión socioeducativa es aquella relativa a la "Planificación del tiempo" con una puntuación de 6.92 .

En cuanto al factor 3 referido a programas socioeducativos con financiación público-privada, los profesionales valoran que deben ser potenciados en diferente medida, dando una mayor valoración a programas más consolidados como el Programa Incorpora -itinerarios personalizados de inserción (Obra Social "La Caixa")- puntuándolo con un 8.24, seguido de los Itinerarios de inserción sociolaboral, con una puntuación de 8.10, tras estos se sitúan, como aquellos programas a potenciar, las Agencias de colocación, valoradas con una puntuación de 8.05. En el siguiente bloque encontramos las Ayudas al emprendimiento con una valoración de 7.36, las Lanzaderas de empleo -búsqueda de trabajo en equipo de forma personalizada 
(Fundación Santa María La Real) - valoradas con un 7.3, Vives Emplea (Acción contra el Hambre) con una puntuación de 6.92 y finalmente Aula Mentor, que obtiene una puntuación de 6.75. Por lo tanto, de aquellos programas emergentes en el ámbito de la inclusión socioeducativa, destaca el Programa Incorpora -Itinerarios personalizados de inserción (Obra Social "La Caixa”)- y del que Longás, De Querol, Ciraso, Rierad y Úcar, 2018 y Gómez, 2019 nos hablan por su impacto positivo en la inserción real de estos jóvenes en situación vulnerable.

\section{DISCUSIÓN Y CONCLUSIONES}

Nuestro trabajo responde a los objetivos que nos habíamos planteado al inicio, conocer los diferentes programas e iniciativas existentes que promueven la inclusión socioeducativa de jóvenes en situación vulnerable e identificar la efectividad de los programas en materia de inclusión socioeducativa y sus áreas de mejora (Salva, Nadal \& Agnés, 2017; Olmos \& Más, 2018; Moreno-Medina \& Vázquez, 2019; García, 2020; Leiva et al. 2020; Alkorta, 2020).

Así mismo, era objeto de este trabajo conocer las iniciativas existentes que promueven la inclusión socioeducativa de jóvenes en situación vulnerable y que combinan formación, competencias clave y prácticas profesionales en empresas para el desarrollo de competencias técnicas que favorezcan la inclusión socioeducativa, lo que converge con los resultados de otros estudios desarrollados en los últimos años en el contexto español (Herrera, 2014; Olmos, 2014; Prieto, 2015; Salva, Nadal \&Agnés, 2017; Olmos \& Más, 2018; Martíns, Carneiro, Campos, Mota, Negrão, Baptista \& Matos, 2020; García 2019). En este sentido, hemos podido conocer el grado de efectividad de los programas financiados con fondos públicos con financiación europea destacando aquellos con mayores fortalezas, como la Formación Profesional para el Empleo y las prácticas profesionales no laborales, lo que supone que las instituciones públicas deben priorizar este tipo de iniciativas y seguir atendiéndolas a través de los diferentes agentes sociocomunitarios (Ainscow, Booth \& Dyson, 2006; Corchuelo, Aránzazu, González \& Morón, 2016; Mcmillan, Rodrik \& Sepúlveda, 2017; Aguilera \& Caballero, 2019; García, 2019). En este punto, no podemos olvidar que estudios realizados en el entorno social y geográfico más cercano también nos apuntan a la necesidad de establecer nuevas fórmulas y diseños formativos y de inserción laboral más holísticos, caracterizados por mayores dosis de creatividad y de participación de entidades sociales y de la iniciativa social (Giaré, Ricciardi \& Borsotto, 2020).

También se han identificado de forma más concreta aquellas competencias técnicas favorecedoras de la inclusión socioeducativa teniendo mayor peso las relacionadas con la "Búsqueda de empleo (orientación y diseño del currículum)” y el “Autoconocimiento y motivación 
personal” y que como señala Domínguez (2019) contribuyen a ofrecer oportunidades a estas personas para la vuelta al proceso de educación a lo largo de la vida y les permite garantizar su inclusión sociolaboral a corto-medio plazo. Además, dentro de la amplia amalgama de personas y colectivos marginalizados y excluidos de las posibilidades de empleo por dificultades y/o disfuncionalidades de los sistemas formativos, nos encontramos con la necesidad de ofrecer mayor visibilidad y ayuda mutua a grupos históricamente discriminados que requieren también respuestas de personalización y orientación ajustada a sus necesidades formativas, identitarias y/o emocionales (Castillo, 2020).

El Informe de Seguimiento de la Educación en el Mundo 2020 "Inclusión y Educación" ha dibujado un panorama global muy complejo para los jóvenes poniendo de manifiesto el devastador impacto que ha supuesto la pandemia COVID-19 para el desarrollo educativo, social y laboral de estos jóvenes tal y como se muestra en nuestro trabajo y que compartimos con las investigaciones realizadas por Ponce de León (2020).

Hay que destacar además que los resultados obtenidos, y como prospectiva determinante para futuras investigaciones, confirman la necesidad de seguir trabajando en programas de estas características, consolidando el derecho a una educación de calidad para estos jóvenes, tal y como propugna la Agenda 2030 y su ODS 4 (2015) y que han sufrido el abandono escolar temprano (Olmos, 2014; Prieto, 2015; Salva, Nadal \& Agnés, 2017; Vázquez \& Barrera, 2017; Olmos y Más, 2018; Martíns, Carneiro, Campos, Mota, Negrão, Baptista \& Matos, 2020; García 2020).

\section{LIMITACIONES}

Este estudio presenta algunas limitaciones que deben ser tenidas en cuenta en el diseño de futuras investigaciones. En primer lugar, los datos obtenidos se basan de forma exclusiva en profesionales de la inclusión socioeducativa si bien, sería acertado contar con las apreciaciones de los propios jóvenes participantes en estos programas y otros agentes, con el fin de comparar las percepciones acerca del impacto y proyección de los procesos formativos llevados a cabo. En segundo lugar, sería útil contar con un marco externo para evaluar las competencias profesionales y que tienen una mayor relación con las demandas empresariales y del mercado laboral, por ejemplo, con datos de observación y autoevaluaciones de las entidades colaboradoras, entidades educativas, sociales y empresas. Futuros estudios deben profundizar en el análisis de nuevas evidencias de cambio en las prácticas formativas y de orientación para la inclusión sociolaboral, así como en el impacto de nuevas propuestas formativas que avancen hacia metodologías más innovadoras que fomenten la cooperación y la justicia social. 
El futuro de la inclusión socioeducativa y laboral requiere la conexión interdisciplinar y transversal de distintas instancias formativas, tanto públicas como privadas interesadas en una proyección formativa y laboral sostenible, con proyección de futuro y con una atención específica y personalizada en aquellos grupos marginalizados que requieren más ayuda, orientación y acompañamiento en sus propios itinerarios de desarrollo personal y profesional (García, Leiva, Junior \& Fontoura, 2021). Propuestas formativas atractivas, innovadoras, creativas y con un compromiso inequívoco por la inclusión y el bienestar social requiere las mejores y mayores sinergias entre ámbito público y privado, combinando conciencia e innovación social en una economía más sostenible y que ofrezca oportunidades de crecimiento y justicia social desde un enfoque de inclusividad.

\section{REFERENCIAS BIBLIOGRÁFICAS}

Aguilera, Francisco \& Caballero, Pablo. (2019), "Actions of social and labour inclusion with ICT: an assessment of the Velez-Malaga urban area”, New Trends and Issues Proceedings on Humanities and Social Sciences, 6(1), 117-125 DOI: https://doi.org/10.18844/prosoc.v6i1.4162

Ainscow, Mel, Booth, Tony \& Dyson, Alan (2006), Improving Schools, Developing Inclusion, Routledge.

Alkorta, Aitor (2020), "La inclusión sociolaboral de los grupos vulnerables. Colectivos y formas de inclusión a través del trabajo", CIRIEC-España, revista jurídica de economía social y cooperativa, (36), 15-53. http://ciriec-revistajuridica.es/wp-content/uploads/comen36-03.pdf

Arredondo, Rafael y Vizcaíno, Deborah (2020), "Fracaso escolar y abandono educativo temprano. Las escuelas de segunda oportunidad como alternativa”, RUMBOS TS, 23, 63-79. DOI: https://doi. org/10.51188/rrts.num23.423

Castillo, Jose Ignacio (2020), “Terapia Ocupacional en la inclusión laboral de personas trans. Un ensayo reflexivo", Revista de Estudiantes de Terapia Ocupacional, 7(2), 11-25. http://www.reto.ubo.cl/ index.php/reto/article/view/97

Comisión de las Comunidades Europeas (1995), "Libro Blanco sobre la Educación y la Formación”, Bruselas. https://eur-lex.europa.eu/LexUriServ/LexUriServ.do?uri=COM:1995:0590:FIN:ES:PDF

Comisión Europea (2018), "Competencias clave para el aprendizaje permanente un marco de referencia europeo. Recomendación del consejo de 22 de mayo de 2018 relativa a las competencias clave para el aprendizaje permanente", Diario Oficial de la Unión Europea (2018/C 189/01). https://eur-lex.europa.eu/legal-content/ES/TXT/PDF/?uri=CELEX:32018H0604(01)\&from=SV \#: :text=Entre \%20las \%20competencias \%20clave \%20se,creatividad \%20y \%20las \%20capacidades \%20interculturales. 
Corchuelo, Celia (2014), "Respuestas educativas del profesorado en la Escuela de Segunda Oportunidad: estudio de caso", Tesis doctoral. http://rabida.uhu.es/dspace/handle/10272/8875

Corchuelo, Celia, Aránzazu, Carmen María, González-Faraco, Juan Carlos, y Morón, Juan Agustín (2016), "Al borde del precipicio: las escuelas de segunda oportunidad, promotoras de inserción social y educativa", International fournal of Educational Research and Innovation (IfERI), 6, 95109. https://www.upo.es/revistas/index.php/IJERI/article/view/1848

Domínguez, Daniel (2019)," Aproximación conceptual y aplicada a la educación permanente", SSRN, 1-22. http://dx.doi.org/10.2139/ssrn.3496021

Escarbajal, Andrés. e Izquierdo, Tomás (2013), "Percepciones psicosociales de la exclusión que determinan la inclusión sociolaboral”, Revista de Ciencias Sociales (RCS). Vol. XIX, No. 1, pp. 13 - 21. file://C:/Users/Usuario/Downloads/Dialnet-PercepcionesPsicosocialesDeLaExclusionQueDetermina-4238716\%20(1).pdf

Escarbajal, Andrés, Izquierdo, Tomás y López, Olivia (2013), "Análisis del bienestar psicológico en grupos en riesgo de exclusión social", Anales de psicología, vol. 30, n. ${ }^{\circ}$ 2, pp. 541-548. DOI: http:// dx.doi.org/10.6018/analesps.30.2.156591

Eurofound and the International Labour Office (2017), "Working anytime, anywhere: The effects on the world of work", Publications Office of the European Union, Luxembourg, and the International Labour Office, Geneva.

European Comission (2020). Digital Education Action Plan 2021-2027, Resetting education and training for the digital age. https://ec.europa.eu/education/sites/default/files/document-library-docs/ deap-communication-sept2020 en.pdf

García, Francisco José (2019), “Inclusión Sociolaboral de personas con especiales dificultades de acceso al empleo”. Belmonte, L.J., Gázquez, J.J., Simón, M.M., Soriano, J.G., Oropesa, N.F., Martos, A. y Barragán, A.B. (Ed.). Innovación Docente e Investigación en Ciencias Sociales y Jurídicas, 237247. Madrid: Dyckinson.

García, Francisco José (2020), "Políticas públicas sobre sostenibilidad e inclusión social en España. Aportaciones desde la investigación científica”, XII Congreso Internacional de Educación Superior. Congreso Universidad 2020, La Habana, Cuba. https://hdl.handle.net/10630/19300

García, María del Mar, Medina, Marta, Amashta, Giselle., e Higueras, Lina (2021), “ Socio-Educational Factors to Promote Educational Inclusion in Higher Education. A Question of Student Achievement", Education Sciences, 11(3), 123. DOI: 10.3390/educsci11030123 
García, Francisco José, Leiva, Juan José, Fontoura, Eduardo, Piccoli, Flaviany (2021), "Inclusión social de mujeres rurales a través de programas de alfabetización digital para el empleo", Revista Complutense de Educación, 32(1), 15-25. DOI: https://doi.org/10.5209/rced.67590

Giaré, Francesca, Ricciardi, Gabriella, y Borsotto, Patrizia (2020), "Migrants workers and processes of social inclusion in Italy: The possibilities offered by social farming", Sustainability, 12(10), 3991. DOI: $\underline{10.3390 / \text { su12103991 }}$

Gómez, María José (2019), “La formación en la lucha contra el empleo pobre en España: un estudio sobre el perfil de los trabajadores participantes en programas para el fomento de la empleabilidad. Instrumentos Normativos para la Mejora de las Trayectorias de los Working Poor y su Inclusión Social (DER2015-63701-C3-1-R)”, Financiado por el Ministerio de Economía y Competitividad y el Fondo Europeo de Desarrollo Regional.https://idus.us.es/bitstream/handle $/ 11441 / 83241 /$ Formaci \%C3 \%B3n \%20en \%20la \%20lucha....pdf?sequence=1

Herrera, Manuel (2014), "Formación y empleo para colectivos vulnerables". Nueva Revista, n. ${ }^{\circ} 149$. https://www.nuevarevista.net/destacados/formacion-y-empleo-para-colectivos-vulnerables/

Leiva, Juan José, Parody, Lucía, Alcalá, María José, Santos, María Jesús, Negri, María Isabel, Fernández, Miguel Ángel, Franco, Pablo, Del Pino, Lorena, Ortega, Sara, Matas, Antonio, Guerrero, José Francisco \& García, Francisco José, "Diseño del diagnóstico de inclusión social, laboral y educativa y digital del proyecto Edusi Bic Vélez”, Investigación y experiencias de innovación pedagógica inclusiva en una sociedad intercultural y en red. En Leiva, J. y Matas, A. (2020) Investigación y experiencias de innovación pedagógica inclusiva en una sociedad intercultural y en red (231-255). Dykinson.

Ley Orgánica 3/2020, de 29 de diciembre, por la que se modifica la Ley Orgánica 2/2006, de 3 de mayo, de Educación. https://www.boe.es/diario_boe/txt.php?id=BOE-A-2020-17264

Longás, Jordi, De Querol Roser, Ciraso, Anna, Rierad, Jordi \& Úcar, Xavier (2018), "Redes de acción socioeducativa contra la pobreza infantil. Evaluación de la percepción de impacto del programa Caixa Proinfancia”, Revista de estudios y experiencias en educación, Número Especial 2, 109-126. http://www.rexe.cl/ojournal/index.php/rexe/article/view/494

Martíns, Filipe, Carneiro, Alexandra, Campos, Luisa. Mota, Luisa, Negrão, Mariana, Baptista, Isabel y Matos, Raquel (2020), "Derecho a una segunda oportunidad: lecciones aprendidas. De la experiencia de quien abandonó y regresó a la educación", Pedagogía Social. Revista Interuniversitaria, 35, 139-153. DOI: 10.7179/PSRI_2020.36.09

Mcmillan, Margaret, Rodrik, Dani \& Sepúlveda, Claudia (2017), Structural Change, Fundamentals and Growth: A Framework and Case Studies, International Food Policy Research Institute Washington, DC.

112 • International Journal of New Education | Núm. 8 
Mills, Martin y Mc Gregor, Glenda (2010), "Re-engaging students in education. Success Factors in alternative schools. Youth affairs network of Queesland Government”, http://www.yanq.org.au/ uploads/1/4/1/7/14174316/yanq report final-art press compress.pdf

Ministerio de Empleo y Seguridad Social (2020), "Programa Operativo de Empleo, Formación y Educación. Fondo Social Europeo", https://www.mites.gob.es/uafse/es/properativos/poefe/index.htm

Moreno-Medina, Irene, y Vázquez, Carmen (2019), "Sinergias entre instituciones socioeducativas como respuesta ante las necesidades sociales. Una aproximación a través del análisis de redes sociales”, International Journal of New Education, 2(1), 85-101. https://revistas.uma.es/index. $\mathrm{php} / \mathrm{NEIJ} /$ article/view/6560

Olmos, Patricia (2014), “Competencias básicas y procesos perceptivos: factores claves en la formación y orientación de los jóvenes en riesgo de exclusión educativa y sociolaboral”, Revista de Investigación Educativa, 32(2), 531-546. http://dx.doi.org/10.6018/rie.32.2.181551

Olmos, Patricia y Mas, Óscar (2014), "Jóvenes, fracaso escolar y escuelas de segunda oportunidad", REOP, 24 (1), 78-93. DOI: https://doi.org/10.5944/reop.vol.24.num.1.2013.11272

Olmos, Patricia y Mas, Óscar (2018), "Validación de AUTOCOM: autoevaluación de las competencias básicas de jóvenes en el marco de programas formativos de segunda oportunidad”, Revista Electrónica de Investigación Educativa, 20(4), 49-61. https://doi.org/10.24320/redie.2018.20.4.1674

Orden PRA/37/2018, de 16 de enero, por la que se aprueban las bases reguladoras para la concesión de las ayudas del Fondo Social Europeo, previstas en el Programa Operativo de Empleo, Formación y Educación, destinadas a entidades locales para la inserción de las personas más vulnerables. https://www.boe.es/diario boe/txt.php?id=BOE-A-2018-896

Ponce de León, Laura (2020), "Reflexiones sobre la COVID 19 y población vulnerable: ¿Estado de Bienestar o Neoliberalismo?”, Ehquidad International Welfare Policies and Social Work fournal, ${ }^{\circ}$ 14, DOI: https://doi.org/10.15257/ehquidad.2020.0010

Prieto, Beatriz (2015), "El camino desde la vulnerabilidad escolar hacia el desenganche educativo. El papel de las escuelas de segunda oportunidad en la estrategia contra el abandono educativo", Revista de Currículum y Formación de Profesorado, 19 (3), 110-125. https://recyt.fecyt.es/index. php/profesorado/article/view/43633

Ruiz, Carlos (2002), Instrumentos de investigación educativa: Procedimientos para su diseño y validación, Barquisimeto. CIDEG (Centro de Investigación y Desarrollo en Educación y Gerencia).

Salva, Francesca, Nadal, Joan y Meliá, María Agnes (2016), "Itinerarios de éxito y rupturas en la educación de segunda oportunidad”, Revista Latinoamericana de Ciencias Sociales, Niñez y Juventud, 14(2), 1405-1419. DOI:10.11600/1692715x.14235251115 
UNESCO (2015), “Declaración de Incheon y Marco de Acción para la realización del Objetivo de Desarrollo Sostenible 4. Hacia una educación inclusiva, equitativa y de calidad y un aprendizaje a lo largo de la vida para todos" https://unesdoc.unesco.org/ark:/48223/pf0000233137 spa

UNESCO (2020), "Informe de seguimiento de la educación en el mundo. Inclusión y educación”, Todos y todas sin excepción. https://es.unesco.org/gem-report/report/2020/inclusion

Unión Europea (2018), "Resolución del Consejo de la Unión Europea y los Representantes de los Gobiernos de los Estados miembros, reunidos en el Consejo, sobre un marco para la cooperación europea en el ámbito de la juventud: la Estrategia de la Unión Europea para la Juventud 2019-2027” https://eur-lex.europa.eu/legal-content/ES/TXT/PDF/?uri=CELEX:42018Y1218(01)\&from=ES

Vázquez, María Dolores (2015), "Estrategias metodológicas formativas para la inserción sociolaboral de jóvenes en riesgo o situación de exclusión social, que provienen del fracaso escolar extremo", Tesis Doctoral. https://www.educacion.gob.es/teseo/imprimirFicheroTesis. do?idFichero=1Ua2LbSiyoM \%3D

Vázquez, María José y Barrera, Evaristo (2017), "Las prácticas de empresa para la inserción sociolaboral de jóvenes con fracaso escolar”, En Anduli, Revista Andaluza de Ciencias Sociales, n¹6. pp. 157-174 https://revistascientificas.us.es/index.php/anduli/article/view/5222/4492 